\title{
Black and Native Overdose Mortality Overtook that of White Individuals During the COVID-19 Pandemic
}

\author{
Joseph Friedman, $\mathrm{MPH}^{1,2}$, Helena Hansen, $\mathrm{MD}, \mathrm{PhD}^{2}$ \\ ${ }^{1}$ Medical Scientist Training Program, University of California, Los Angeles \\ ${ }^{2}$ Center for Social Medicine and Humanities, University of California, Los Angeles
}

\begin{abstract}
Drug overdose mortality rates have increased sharply during the COVID-19 pandemic. In recent years, overdose death rates were rising most rapidly among racial/ethnic minority communities. The pandemic has disproportionately affected communities of color in a wide swath of health, social, and economic outcomes. Careful attention is therefore warranted to trends in overdose mortality by race/ethnicity during COVID-19. We calculated total drug overdose death rates per 100,000 population by race/ethnicity for the 1999-2020 time period. We find that Black overdose mortality overtook that of White individuals in 2020 for the first time since 1999. Between 2019 and 2020 Black individuals had the largest percent increase in overdose mortality, of $48.8 \%$, compared to $26.3 \%$ among White individuals. In 2020 , Black overdose death rates rose to 36.8 per 100,000 , representing $16.3 \%$ higher than the rate for White individuals for the same period. American Indian and Alaska Native (AI/AN) individuals experienced the highest rate of overdose mortality in 2020, of 41.4 per 100,000 , representing $30.8 \%$ higher than the rate among White individuals. Our findings suggest that drug overdose mortality is increasingly becoming a racial justice issue in the United States and appears to have been exacerbated by the COVID-19 pandemic. Providing individuals with a safer supply of drugs, closing gaps in access to MOUD and harm reductions services, and ending routine incarceration of individuals with substance use disorders represent urgently needed, evidencebased strategies that can be employed to reduce rising inequalities in overdose.
\end{abstract}


44 Drug overdose mortality rates have increased sharply during the COVID-19 pandemic ${ }^{1}$. In recent

45 years, overdose death rates were rising most rapidly among racial/ethnic minority

47 swath of health, social, and economic outcomes. Careful attention is therefore warranted to

48 trends in overdose mortality by race/ethnicity during COVID-19.

\section{Methods}

We calculated total drug overdose death rates per 100,000 population by race/ethnicity for the 1999-2020 time period, using data obtained from the National Center for Health Statistics ${ }^{3}$.

52 Records from 2020 were provisional and may underestimate the final level of drug overdose 53 related mortality. Drug overdose deaths were classified as those assigned with the underlying

54 cause of death in the ICD-10 categories pertaining to unintentional, suicide, homicide, or

55 undetermined intent drug overdose deaths, (X40-44, X60-64, X85, or Y10-14, respectively). We

56 calculated annual percent change in a race/ethnicity specific fashion for the 2000-2020 period.

57 All analyses were conducted using R version 4.0.3. See appendix for more details.

\section{Results}

We find that Black overdose mortality overtook that of White individuals in 2020 for the first

60 time since 1999. In 2020, Black overdose death rates rose to 36.8 per 100,000, representing

$61 \quad 16.3 \%$ higher than the rate for White individuals for the same period. This is a sharp reversal of

62 the Black-White overdose mortality gap noted in 2010, when the rate among White individuals was double (100.1\% higher) than that seen among Black individuals. These shifts reflect that 
64 Black communities have experienced higher annual percent increases in overdose deaths

65 compared to their White counterparts each year since 2012. In 2020 Black individuals had the

66 largest percent increase in overdose mortality, of $48.8 \%$, compared to $26.3 \%$ among White

67 individuals.

68 American Indian and Alaska Native (AI/AN) individuals experienced the highest rate of overdose

69 mortality in 2020 , of 41.4 per 100,000 , representing $30.8 \%$ higher than the rate among White

70 individuals. Between 1999 and $2017 \mathrm{Al} / \mathrm{AN}$ overdose mortality rates were close to those

71 experienced by White individuals, with rates among Al/AN first overtaking those of Whites by a

72 large margin in 2019. In 2020 overdose mortality increased by $43.3 \%$ for $\mathrm{Al} / \mathrm{AN}$ individuals.

73 Drug overdose rates among Latinx individuals remained the lowest among the 4 race/ethnicity

74 groups assessed throughout the study period, however they also experienced a large percent

75 increase in 2020 of $40.1 \%$.

76 For all race/ethnicity groups assessed, the relative increases observed in 2020 were higher than

77 any prior percent change observed between 1999-2019.

\section{Discussion}

79 The overdose crisis in the United States is increasingly driven by a toxic illicit drug supply

80 characterized by polysubstance use of potent synthetic opioids and benzodiazepines, as well as

81 high-purity methamphetamine. The high-and unpredictably variable-potency of the illicit

82 drug supply may be disproportionately harming communities of color for various reasons.

83 Deep-seated inequalities in living conditions including stable housing and employment, policing 
84 and arrests, preventive care, harm reduction, telehealth, medications for opioid use disorder

85 (MOUD) and naloxone, are likely playing a key role ${ }^{4,5}$.

86 Further, the increasing toxicity of the drug supply has increased the lethality of recent

87 incarceration - which disproportionately affects Black and Native individuals due to structural

88 racism in the criminal justice system - as a risk factor for overdose mortality. Recently

89 incarcerated individuals have reduced opioid tolerance and less knowledge of shifts in drug

90 potency 6.

91 Drug overdose mortality is increasingly becoming a racial justice issue in the United States and

92 appears to have been exacerbated by the COVID-19 pandemic. Providing individuals with a

93 safer supply of drugs, closing gaps in access to MOUD, health care, and harm reduction

94 services, and ending routine incarceration of individuals with substance use disorders represent

95 urgently needed, evidence-based strategies that can be employed to reduce rising inequalities

96 in overdose.

97

98

99

100

101

102

103 
medRxiv preprint doi: https://doi.org/10.1101/2021.11.02.21265668; this version posted November 3, 2021. The copyright holder for this preprint (which was not certified by peer review) is the author/funder, who has granted medRxiv a license to display the preprint in perpetuity.

It is made available under a CC-BY-NC-ND 4.0 International license .

Drug Overdose Mortality By Race/Ethnicity, 1999-2020

American Indian or Alaska Native, Non-Hispanic Black or African American, Non-Hispanic Latinx White, Non-Hispanic

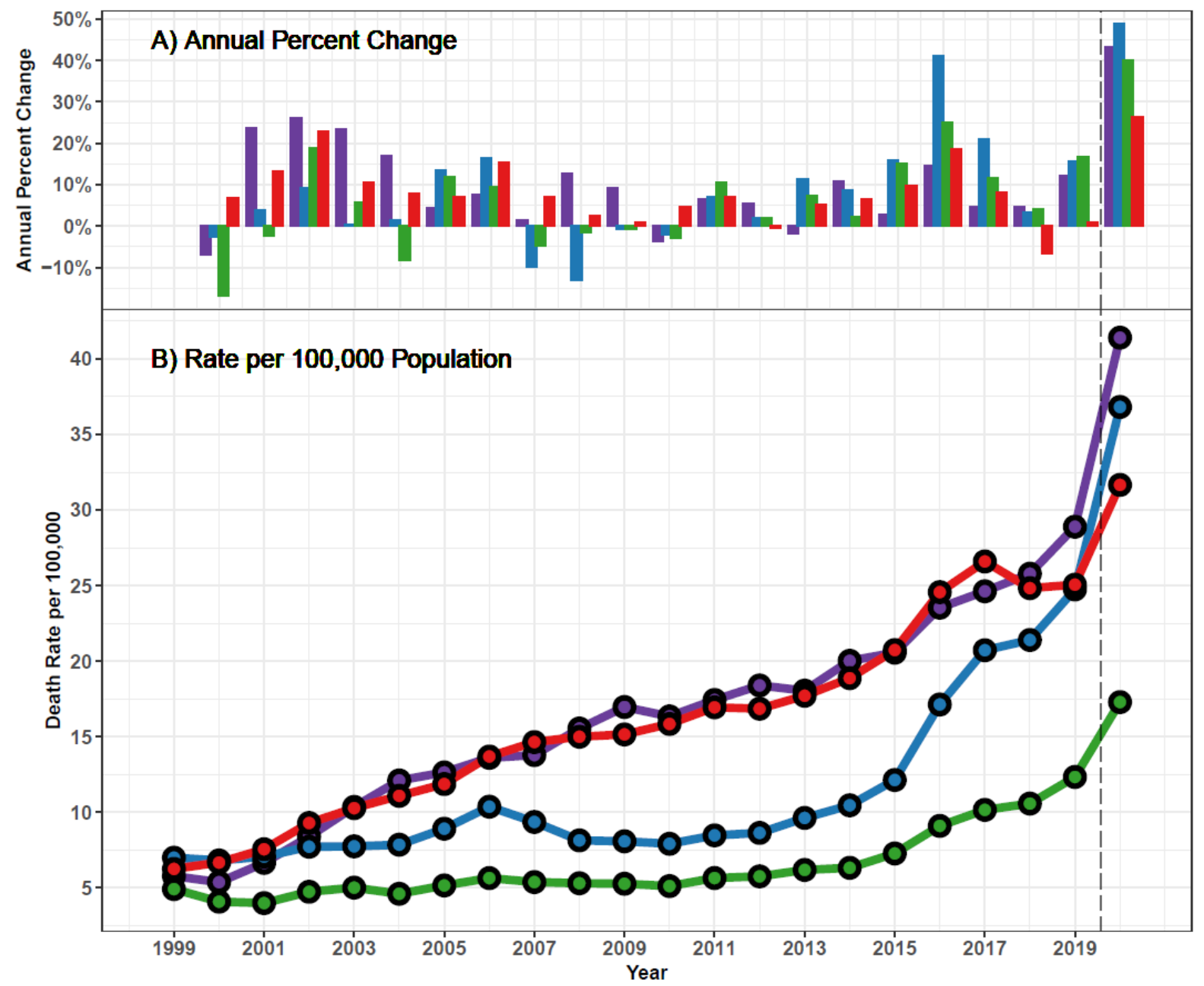

Figure 1. Drug Overdose Mortality by Race/Ethnicity, 1999-2020

Year-to-year percent change in drug overdose mortality by race/ethnicity (top). Drug overdose mortality per 100,000 population by race/ethnicity (bottom). A vertical dashed line separates the COVID-19 pandemic period from prior trends. 
medRxiv preprint doi: https://doi.org/10.1101/2021.11.02.21265668; this version posted November 3, 2021. The copyright holder for this preprint (which was not certified by peer review) is the author/funder, who has granted medRxiv a license to display the preprint in perpetuity.

It is made available under a CC-BY-NC-ND 4.0 International license .

\section{References}

1. Friedman J, Akre S. COVID-19 and the Drug Overdose Crisis: Uncovering the Deadliest Months in the United States, January-July 2020. Am J Public Health. Published online April 15, 2021:e1-e8. doi:10.2105/AJPH.2021.306256

2. James K, Jordan A. The Opioid Crisis in Black Communities. J Law Med Ethics. 2018;46(2):404-421. doi:10.1177/1073110518782949

3. National Center for Health Statistics. Provisional Drug Overdose Deaths by Quarter and Demographics - 2019 to 2020. Published online 2021. Accessed October 23, 2021. https://www.cdc.gov/nchs/data/health_policy/Provisional-Drug-Overdose-Deaths-by-Quarter-andDemographic-Characteristics-2019-to-2020.pdf

4. Lagisetty PA, Ross R, Bohnert A, Clay M, Maust DT. Buprenorphine Treatment Divide by Race/Ethnicity and Payment. JAMA Psychiatry. 2019;76(9):979-981. doi:10.1001/jamapsychiatry.2019.0876

5. Park JN, Rouhani S, Beletsky L, Vincent L, Saloner B, Sherman SG. Situating the Continuum of Overdose Risk in the Social Determinants of Health: A New Conceptual Framework. The Milbank Quarterly. 2020;98(3):700-746. doi:10.1111/1468-0009.12470

6. Brinkley-Rubinstein L, Macmadu A, Marshall BDL, et al. Risk of fentanyl-involved overdose among those with past year incarceration: Findings from a recent outbreak in 2014 and 2015. Drug and Alcohol Dependence. 2018;185:189-191. doi:10.1016/j.drugalcdep.2017.12.014 


\section{Methods Appendix}

\section{Data Preparation and Definitions}

1. Total drug overdose mortality counts for the 1999-2019 period were obtained from the CDC WONDER platform (https://wonder.cdc.gov/controller/saved/D76/D243F427) stratified by race, ethnicity, and year of occurrence. Both population counts, and counts of overdose fatalities were provided, and used as the denominator and numerator, respectively.

2. Overdose mortality rates for $\mathbf{2 0 2 0}$ were calculated using provisional race/ethnicity stratified drug overdose mortality counts released by the National Center for Health Statistics (https://www.cdc.gov/nchs/data/health policy/Provisional-Drug-Overdose-Deaths-by-Quarterand-Demographic-Characteristics-2019-to-2020.pdf).

3. Mid-year population estimates by race/ethnicity for the year 2020 were obtained from the CDC Wonder platform (https://wonder.cdc.gov/single-race-single-year-v2020.html).

4. Total drug overdoses were defined by the following ICD-10 codes:

\begin{tabular}{|l|}
\hline X40 (Accidental poisoning by and exposure to nonopioid analgesics, antipyretics and antirheumatics) \\
\hline X41 (Accidental poisoning by and exposure to antiepileptic, sedative-hypnotic, antiparkinsonism and psychotropic drugs, not elsewhere classified) \\
\hline X42 (Accidental poisoning by and exposure to narcotics and psychodysleptics [hallucinogens], not elsewhere classified) \\
\hline X43 (Accidental poisoning by and exposure to other drugs acting on the autonomic nervous system) \\
\hline X44 (Accidental poisoning by and exposure to other and unspecified drugs, medicaments and biological substances) \\
\hline X60 (Intentional self-poisoning by and exposure to nonopioid analgesics, antipyretics and antirheumatics) \\
\hline X61 (Intentional self-poisoning by and exposure to antiepileptic, sedative-hypnotic, antiparkinsonism and psychotropic drugs, not elsewhere classified) \\
\hline X62 (Intentional self-poisoning by and exposure to narcotics and psychodysleptics [hallucinogens], not elsewhere classified) \\
\hline X63 (Intentional self-poisoning by and exposure to other drugs acting on the autonomic nervous system) \\
\hline X64 (Intentional self-poisoning by and exposure to other and unspecified drugs, medicaments and biological substances) \\
\hline X85 (Assault by drugs, medicaments and biological substances) \\
\hline Y10 (Poisoning by and exposure to nonopioid analgesics, antipyretics and antirheumatics, undetermined intent) \\
\hline Y11 (Poisoning by and exposure to antiepileptic, sedative-hypnotic, antiparkinsonism and psychotropic drugs, not elsewhere classified, undetermined intent) \\
\hline Y12 (Poisoning by and exposure to narcotics and psychodysleptics [hallucinogens], not elsewhere classified, undetermined intent) \\
\hline Y13 (Poisoning by and exposure to other drugs acting on the autonomic nervous system, undetermined intent) \\
\hline Y14 (Poisoning by and exposure to other and unspecified drugs, medicaments and biological substances, undetermined intent) \\
\hline
\end{tabular}

5. 'Latinx' individuals were defined as any persons for which ethnicity was defined as Hispanic or Latino, regardless of race. "American Indian or Alaska Native, Non-Hispanic," "Black or African American, Non-Hispanic," and "White, Non-Hispanic" individuals were defined as individuals of each race, who had their ethnicity listed as "non-Hispanic."

6. Rates were calculated as total drug overdose deaths per 100,000 population, according to midyear population.

7. Year-to-year percent change values were calculated for each year $t$ in a race/ethnicity groupspecific fashion according to the following formula:

((Overdose Death Rate /Overdose Death Rate $\left.\left._{t-1}\right)-1\right) * 100 \%$.

8. Data were visualized using $R$ version 4.0.3.

Methodological Consideration

1. Records from 2020 were provisional and may underestimate the final level of drug overdose related mortality.

2. Race/ethnicity may be incorrectly assigned in some overdose deaths. This is a known issue especially for Native individuals. 
medRxiv preprint doi: https://doi.org/10.1101/2021.11.02.21265668; this version posted November 3, 2021. The copyright holder for this preprint

(which was not certified by peer review) is the author/funder, who has granted medRxiv a license to display the preprint in perpetuity.

It is made available under a CC-BY-NC-ND 4.0 International license.

3. It was not possible to age-standardize the race/ethnicity stratified results given the format of the provisional records from 2020. This is an important area of consideration for future work.

191

4. This work is descriptive, not causal in nature, and it was not possible to show causally what percent of increases in overdose death rates between 2019 and 2020 stem from the COVID-19 pandemic directly. 